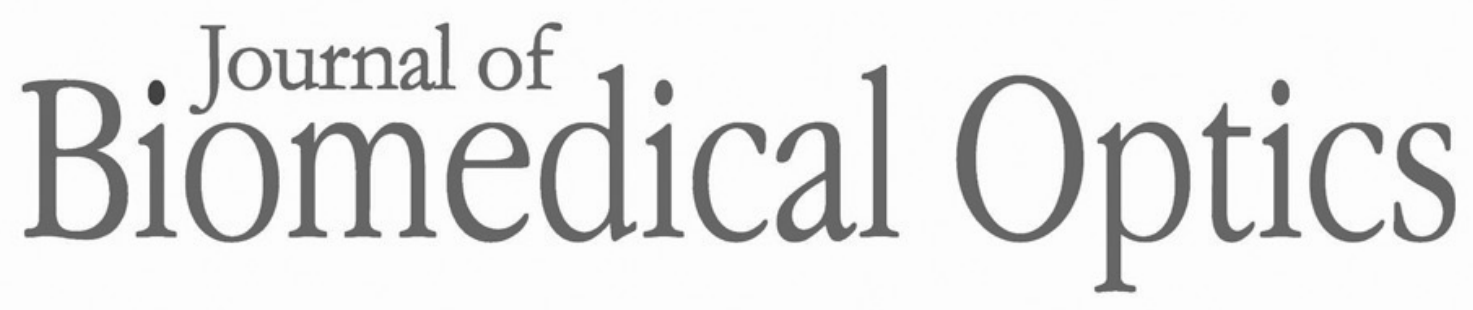

\title{
Report on fluorescence lifetime imaging using multiphoton laser scanning microscopy targeting sentinel lymph node diagnostics
}

Jeemol James

Despoina Kantere

Jonas Enger

Jan Siarov

Ann Marie Wennberg

Marica B. Ericson 


\title{
Report on fluorescence lifetime imaging using multiphoton laser scanning microscopy targeting sentinel lymph node diagnostics
}

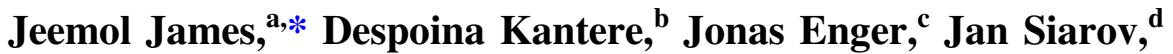 \\ Ann Marie Wennberg, ${ }^{b}$ and Marica B. Ericson ${ }^{a}, *$ \\ ${ }^{a}$ University of Gothenburg, Biomedical Photonics Group, Department of Chemistry \\ and Molecular Biology, Gothenburg, Sweden \\ ${ }^{b}$ University of Gothenburg, Institute of Clinical Sciences, Department of Dermatology \\ and Venereology, Gothenburg, Sweden \\ ${ }^{c}$ University of Gothenburg, Department of Physics, Gothenburg, Sweden \\ ${ }^{\mathrm{d}}$ University of Gothenburg, Department of Pathology, Gothenburg, Sweden
}

\begin{abstract}
Significance: Sentinel lymph node (SLN) biopsy is an important method for metastasis staging in, e.g., patients with malignant melanoma. Tools enabling prompt histopathological analysis are expected to facilitate diagnostics; optical technologies are explored for this purpose.

Aim: The objective of this exploratory study was to investigate the potential of adopting multiphoton laser scanning microscopy (MPM) together with fluorescence lifetime analysis (FLIM) for the examination of lymph node (LN) tissue ex vivo.

Approach: Five LN tissue samples (three metastasis positive and two negative) were acquired from a biobank comprising tissues from melanoma patients. Tissues were deparaffinized and subjected to MPM-FLIM using an experimental MPM set-up equipped with a time correlated single photon counting module enabling FLIM.

Results: The data confirm that morphological features similar to conventional histology were observed. In addition, FLIM analysis revealed elevated morphological contrast, particularly for discriminating between metastatic cells, lymphocytes, and erythrocytes.

Conclusions: Taken together, the results from this investigation show promise for adopting MPM-FLIM in the context of SLN diagnostics and encourage further translational studies on fresh tissue samples.

(C) The Authors. Published by SPIE under a Creative Commons Attribution 4.0 Unported License. Distribution or reproduction of this work in whole or in part requires full attribution of the original publication, including its DOI. [DOI: 10.1117/1.JBO.25.7.071204]
\end{abstract}

Keywords: malignant melanoma; sentinel lymph node; multiphoton microscopy; fluorescence lifetime imaging; autofluorescence.

Paper 190344SSRR received Sep. 27, 2019; accepted for publication Feb. 7, 2020; published online Mar. 14, 2020.

\section{Introduction}

Recent advancements in optical techniques such as multiphoton laser scanning microscopy (MPM),${ }^{1,2}$ confocal reflectance microscopy ${ }^{3}$ and optical coherence tomography ${ }^{4}$ have strengthened ongoing research targeting the development of optical tools that enable intravital tissue biopsy. ${ }^{5,6}$ MPM has been explored in the field of skin cancer research for the last few years and has demonstrated potential for use in facilitating diagnostics of primary malignant melanoma. ${ }^{7,8}$ Fluorescence lifetime imaging microscopy (FLIM) is a time-correlated fluorescence technique that generates images based on the fluorescence lifetime rather than intensity. ${ }^{9-11}$ FLIM has been applied to mapping the local environment of fluorophores such as ion concentration and $\mathrm{pH}$

*Address all correspondence to Jeemol James, E-mail: jeemol.james@gu.se; Marica B. Ericson, E-mail: marica.ericson@gu.se 
sensing in various samples, since fluorescence lifetime depends on the molecular environment. ${ }^{12,13}$ Furthermore, FLIM can enable studies of metabolic activities in cellular environments by identifying the bound and unbound forms of nicotinamide adenine dinucleotide (NADH) and flavin adenine dinucleotide (FAD), two of the most common metabolic markers. ${ }^{14,15}$

According to the Global Burden of Disease Study in $2015,{ }^{16}$ malignant melanoma processes a global incidence of 0.3 million, making it one of the most widely spreading skin cancers across the world. Sweden has the fourth highest number of melanoma incidents out of the 195 countries included in said study. Because of the high metastasizing potential of melanoma, early diagnostics are of utmost importance, and the five-year survival rate is more than $90 \%$ if melanoma is diagnosed in the early stage. ${ }^{17}$ The current clinical diagnostic practice for melanoma is to perform surgical excisions of the primary tumor. In the case of thick melanomas, it is followed by histopathological analysis of the sentinel lymph node (SLN), which is the first lymph node (LN) ascended by the metastatic cells. ${ }^{18-20}$ The histopathological analysis of the LN is timeconsuming and requires arduous laboratory work. The accuracy of the SLN biopsy is not $100 \%$; an erroneous diagnosis is inevitable. ${ }^{18,21,22}$ In addition, the removal of the LNs can lead to several complications such as wound infection, lymphedema, and hematoma. ${ }^{23,24}$ Therefore, the development of a technique that can assist surgeons in early metastasis staging is important. Until now, only a limited number of studies have investigated MPM for the purpose of melanoma metastasis diagnostics in LNs. In a recent study by our group, we observed that morphological features characteristic to melanoma metastasis can be discerned in LN tissues using MPM, ${ }^{25}$ but improved contrast would be required to facilitate diagnostics. Inspired by this work, we propose employing MPM combined with FLIM for investigation of primary melanoma lesions. ${ }^{8,26,27}$

The objective of this work was to investigate the potential for adopting MPM-FLIM to examine melanoma metastasis in SLN tissue. This was done by investigating differences between metastasized and nonmetastasized SLN tissue using MPM-FLIM ex vivo. The fluorescence lifetime from tissue inherent fluorophores was visualized and correlated with histopathological morphological features. To the best of the authors' knowledge this is the first study investigating melanoma metastasis in human SLN tissue ex vivo using MPM-FLIM.

\section{Methods and Materials}

\subsection{Sentinel Lymph Node Tissues}

In total, five $\mathrm{LN}$ tissue samples, three melanoma metastasis positive and two negative, were obtained from a biobank at the Department of Pathology, Sahlgrenska University Hospital, Gothenburg, Sweden, as a part of an ongoing study approved by the local ethics committee (University of Gothenburg, No 145-16). Detailed descriptions of samples and patient demography are provided (see Supplementary Material). Half of the intact tissue blocks were deparaffinized according to a customized protocol (see Supplementary Material) and stored in ethanol (70\%, Sigma Aldrich) at room temperature to hydrate the samples. The other halves of the tissue blocks were processed for ordinary histopathological analysis. The deparaffinized tissues were mounted in customized imaging chambers, using ultrasound transmission gel $(n=1.33$, Parker laboratories) as the immersion media, before subjecting to MPM-FLIM.

\subsection{Histopathological Analysis}

Histopathological analysis of corresponding hematoxylin-eosin (H\&E) stained glass slides was performed using a pathology slide scanner (Oncotopix scan, nanoZoomer, Visiopharm).

\subsection{MPM-FLIM Imaging and Data Analysis}

The experimental MPM set-up is schematically illustrated in Fig. 1. Excitation was obtained by a fs-pulsed ( $\sim 80 \mathrm{fs}$ ) tunable (700 to $900 \mathrm{~nm}$ ) Ti:Sapphire laser (Tsunami, Spectra physics) pumped by a frequency doubled Nd:YAG laser (Millenia, Spectra Physics, $532 \mathrm{~nm}$ ). The output laser power was modulated by a Pockels cell (350-80LA, ConOptics). FLIM was recorded using 


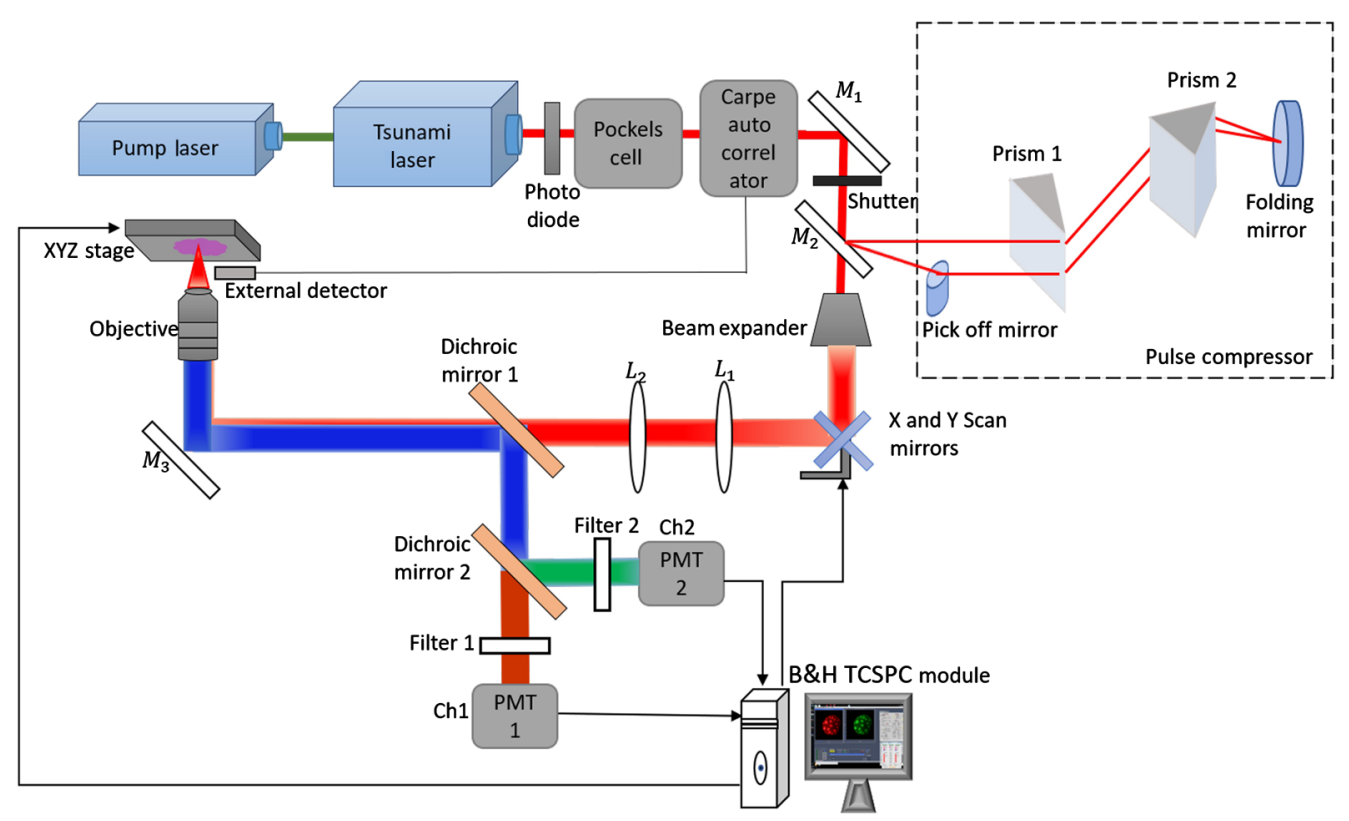

Fig. 1 Schematic diagram of the experimental MPM set-up equipped with Carpe autocorrelator and femtocontrol pulse compressor for FLIM imaging.

an excitation wavelength of $780 \mathrm{~nm}$, and average laser power $<15 \mathrm{~mW}$ and $100 \mathrm{fs}$ pulse duration at the sample plane were maintained by applying an autocorrelator (CARPE, APE) and pulse compressor (Femto control kit, APE).

As an objective lens, a water immersive C "Achroplan" NIR, $40 \times / 0.8 \mathrm{~W}$ (Carl Zeiss) was utilized. The emission signal was detected by two GaAsP detectors (H7422P-40 MOD, Hamamatsu), interfaced to time correlated single photon counting modules SPC 150 (TCSPC, Becker\&Hickl). Two spectral detection channels were set-up using a dichroic mirror $(550 \mathrm{~nm}$ cut off, Semrock Inc.) combined with two filters 580/150 nm and 525/50 nm (Semrock, Brightline), enabling the two channels 1 (red, 550 to $655 \mathrm{~nm}$ ) and 2 (green, 500 to $550 \mathrm{~nm}$ ).

FLIM images were acquired at an area of $512 \times 512$ pixels, using pixel dwell time $1.8 \mu \mathrm{s}$ and scanning a frame at a speed of $0.616 \mathrm{~s}$. Total acquisition time for each image was around $60 \mathrm{~s}$, including multiple frame scans, to increase the number of photons, which improved signal-to-noise ratio. FLIM analysis was performed using SPCImage 8 software (SPCM, Becker\&Hickl) ${ }^{28} \mathrm{~A}$ single exponential decay model was applied to fit the fluorescence lifetime decays. FLIM data from different regions of interest were extracted from SPCImage, and the lifetime histograms were plotted in MATLAB (MathWorks Inc.).

\section{Results}

The results from this exploratory study report on the data acquired using MPM-FLIM ex vivo investigating five $\mathrm{LN}$ tissue samples, three melanoma metastasis positive and two negative. The data from all samples are presented in Figs. S1-S3 in the Supplementary Material. Figure 2 shows representative MPM intensity and FLIM images acquired from one of the melanoma positive tissues (LN1). Included in the figure is the corresponding image of an H\&E stained tissue section from the same sample. As observed from this figure, the MPM intensity image [Fig. 2(b)] and FLIM images [Fig. 2(c), channel 1: 580/150 nm and Fig. 2(d), channel 2: $525 / 50 \mathrm{~nm}$, respectively] display pleomorphism, i.e., varying cell sizes, similar to the H\&E image [Fig. 2(a)]. Similar features were observed in all metastasis positive samples (Figs. S1 and S2 in the Supplementary Material). Overall, the values of the fluorescence lifetimes acquired from the samples are distributed around 100 to 3000 ps as shown in Fig. 2(f). The acquired lifetimes in both spectral channels most likely correspond to signals from NADH and FAD, but they are difficult to separate due to spectral cross-talk. ${ }^{29}$ The long fluorescence lifetime 


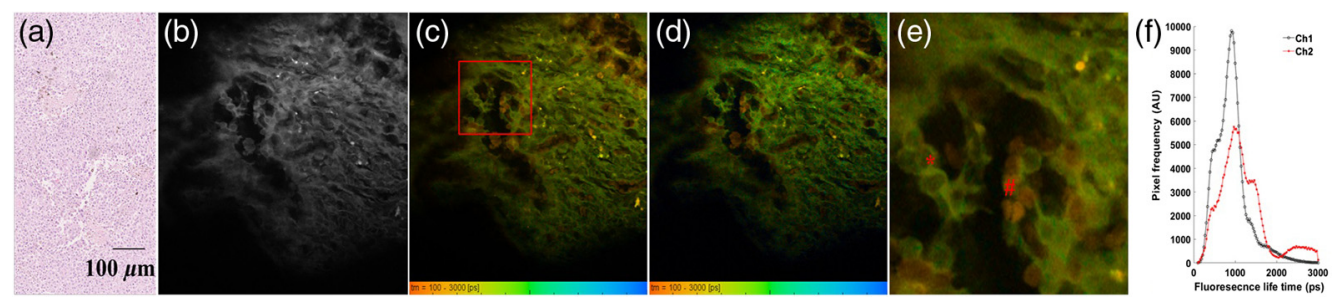

Fig. 2 H\&E stained histologic section (a) together with MPM intensity image (b); FLIM images obtained from channel 1,580/150 nm (c), channel 2, 525/50 nm (d), of SLN (LN1) positive for melanoma metastasis and corresponding fluorescence lifetime distribution histogram (f) obtained from two spectral channels. The zoomed FLIM image (e) corresponding to the region of interest (highlighted red box) in (c). Atypical cells possibly undergoing mitosis $\left(^{*}\right)$ and randomly distributed cells (\#) with short fluorescence lifetime ( 600 ps) are highlighted in (e). MPM and FLIM data acquired using $780 \mathrm{~nm}$ excitation. Field of view: (b)-(d) $\sim 250 \times 250 \mu \mathrm{m}$ and (e) $70 \times 70 \mu \mathrm{m}$. False color scale lifetime data, 256-time channels, ranging from 100 to 3000 ps.

distribution $>2000$ ps in channel 2 [Fig. 2(f)] most likely corresponds to FAD, while the lifetimes in the range 300 to $2000 \mathrm{ps}$ probably originate from NADH,${ }^{29-31}$ which is also observed in channel 1. In the zoomed-in image [Fig. 2(e)], the cell structure becomes more evident; large atypical malignant cells $(\sim 10 \mu \mathrm{m})$ display a fluorescent cytoplasm surrounding a prominent and less fluorescent nuclei. Highlighted in Fig. 2(e) is a possible mitosis (*), thus, supporting the presence of melanoma metastasis. In addition, cells exhibiting significantly shorter lifetime values $\sim 600$ to $700 \mathrm{ps}$ were also discerned, which can be seen in the orange color scale highlighted by (\#). Based on their structure lacking visible nuclei, these cells most likely correspond to erythrocytes, which is further supported by findings discussed below.

As a control, two melanoma negative SLN tissues (LN3 and LN5) were investigated (Fig. S3 in the Supplementary Material). The data presented in Fig. 3 demonstrate representative MPMFLIM data together with a corresponding H\&E stained tissue section of one of the melanoma negative SLN tissues (LN3). When comparing Figs. 2 and 3, it is evident that there is a clear difference in morphological structures between the samples. In the negative control sample, a more homogenous tissue matrix is visible, with hardly discernable small cells $(\sim 7 \mu \mathrm{m})$ most likely representing lymphocytes, as represented in the corresponding H\&E stained section. Interestingly, the distinct bimodal fluorescence lifetime distribution most likely corresponds to NADH and FAD in the two different spectral channels; the short lifetime corresponds to NADH free and the long lifetime probably conforms to both bound NADH and FAD. ${ }^{29,31}$ It is obvious that, when comparing the fluorescence lifetime data, the bimodal distribution is not present to the same extent in the malignant tissue (Fig. 2 and Fig. S2 in the Supplementary Material). Furthermore, the distribution between shorter and longer lifetime components is different in the two spectral channels. Thus the comparison of the lifetime distributions in the two different channels can potentially add spectral information to the morphological interpretation, thus facilitating diagnostics.

The data in Fig. 4 represent MPM and FLIM images obtained from the metastasis positive LN2 sample, but from a different morphological region. As seen from the MPM intensity image

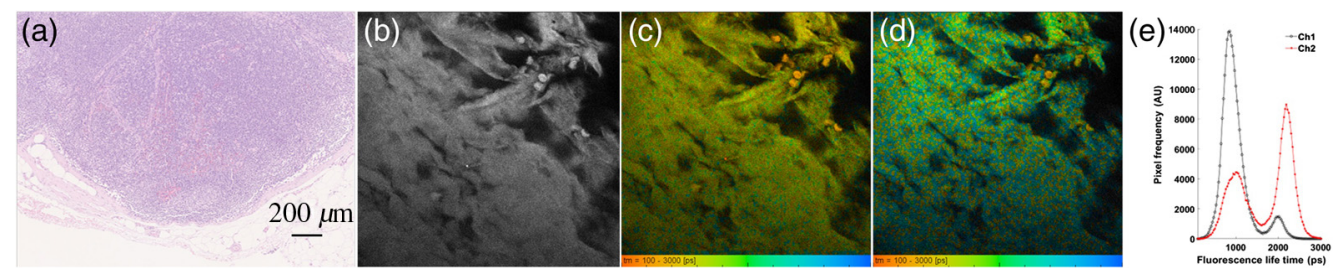

Fig. 3 H\&E stained histologic section (a) together with MPM intensity (b); FLIM images obtained from channel $1,580 / 150 \mathrm{~nm}$ (c), channel 2, 525/50 nm (d) of a melanoma metastasis negative SLN (LN3) and fluorescence lifetime distribution histogram (e) obtained from both spectral channels. MPM and FLIM data acquired using $780 \mathrm{~nm}$ excitation. Field of view: (b)-(d) $\sim 350 \times 350 \mu \mathrm{m}$. False color scale fluorescence lifetime data, 256-time channels, ranging from 100 to 3000 ps. 

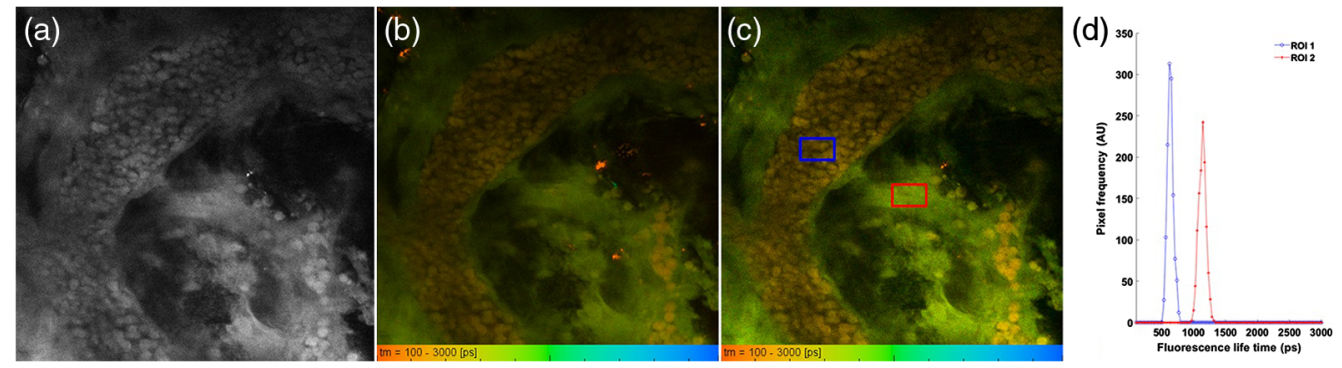

Fig. 4 MPM intensity (a), FLIM images obtained from channel 1,580/150 nm (b), and channel 2 , $525 / 50 \mathrm{~nm}$ (c), of a positive SLN sample (LN2) and fluorescence lifetime distribution (d) obtained from different regions of interest as highlighted in (c). Field of view: $350 \times 350 \mu \mathrm{m}$. False color scale fluorescence lifetime data, 256-time channels, ranging from 100 to 3000 ps. MPM and FLIM data are acquired at $780 \mathrm{~nm}$.

[Fig. 4(a)], the morphological features are difficult to discern. However, turning to the FLIM images obtained from both spectral channels [Figs. 4(b) and 4(c)], completely different structures are revealed based on the lifetime. A structure of bright cells without clear nuclei and exhibiting short fluorescence lifetimes ( $600 \mathrm{ps})$ are clearly discerned in orange-red color. As this structure resembles a blood vessel, the interpretation is that the cells appearing in the orange-red color scale probably correspond to erythrocytes. This is more evident in Fig. 4(d), where the fluorescence lifetime distribution from the two different regions of interest is shown. The fluorescence lifetime distribution from the blood vessel (blue square) is centered around 500 to $800 \mathrm{ps}$, which is similar to the fast fluorescence decay of red blood cells as reported in the literature. ${ }^{32,33}$ By contrast, the lifetime distribution from the metastatic tissue region (red square) has 1000 to 1500 ps, which is analogous to the histogram from Fig. 2. Overall, these results indicate the potential of MPM-FLIM images to visualize the morphological features of positive and negative metastasized SLN tissue samples with better contrast features alongside the fluorescence lifetime information.

\section{Discussion and Conclusion}

Tools are required that can facilitate and complement histopathological analyses of LN tissues, particularly in connection to SLN diagnostics for cancer management, such as early metastatic staging of malignant melanoma. Recent work by our group has demonstrated the potential for employing MPM for investigating SLN tissue ex vivo, ${ }^{25}$ based on extracting LN tissue from a tissue biobank comprising tissues from melanoma patients having undergone SLN surgery at the local hospital. This biobank serves as a unique resource for exploratory studies aimed at the development of technologies targeting clinical translation. It was found that morphological features observed using MPM correlate with histology; however, further means to improve contrast are desirable. Thus, in this paper, we have extended these investigations to include FLIM analysis, using tissues from the same biobank. The intact tissue blocks were deparaffinized and subjected to MPM-FLIM ex vivo using an experimental MPM platform, complementary to the previous commercial MPM utilized in the previous work. ${ }^{25}$ Excitation was performed at $780 \mathrm{~nm}$, and the emission was collected in red channel (ch1, 550 to $655 \mathrm{~nm})$ and green channel (ch2, 500 to $550 \mathrm{~nm}$ ), corresponding to the autofluorescence wavelength region expected for endogenous tissue chromophores, e.g., primarily NADH and FAD. ${ }^{29-31}$ In agreement with our previous study, morphological features signifying metastatic as well as normal lymphatic tissue were identified; however, contrast was enhanced and observed by highlighting morphologic features based on differences in fluorescence lifetime.

The bimodal lifetime distribution observed in the control (i.e., nonmetastatic) tissue samples was most likely interpreted in the context of free NADH and is reported to have a shorter lifetime ( $\sim 300 \mathrm{ps})$, and the bound NADH exhibit a longer lifetime ( 2000 to $2300 \mathrm{ps}){ }^{30,31,34}$ In this study, the lifetime peaks were found around 1000 to 2300 ps (Fig. 3). Since the longer lifetime was more prominent in the green channel (channel 2), the signal most likely also comes from FAD, which is reported to have a lifetime $\sim 3000$ ps. ${ }^{15}$ The shift in lifetimes for free NADH and 
FAD is most likely attributed to the fact that the investigated tissue has undergone substantial work-up by the preceding fixation and deparaffination process. It should be noted that the observed lifetime distribution of NADH and FAD is in agreement with earlier reports demonstrating increasing lifetimes ( $\sim 300$ to $400 \mathrm{ps}$ ) in fixed tissues due to the different fixation protocols and mounting media. ${ }^{35,36}$

Also evident from the data was the identification of erythrocytes and blood vessels exhibiting short lifetimes $\sim 500$ to $600 \mathrm{ps}$ in the red channel and $\sim 600$ to $800 \mathrm{ps}$ in the green channel. It is reported that pure forms of haemoglobin and red blood cells in tissues have fast fluorescence decay $(\sim 300 \mathrm{ps}){ }^{32,33}$ However, contrary to these findings, a recent report on multiphoton excited haemoglobin in stored blood revealed a long-lifetime $(\sim 1300 \mathrm{ps})$ component in addition to a short lifetime value $(\sim 280 \mathrm{ps}) .^{37}$ The fluorescence lifetime values from our study are slightly higher than the values reported previously in the literature. This discrepancy could potentially be related to the metastasis microenvironment of the $\mathrm{LN}$ tissue, but it is most likely related to the preceding fixation and work-up of the tissue. Thus these discrepancies in lifetimes point to the necessity of confirming these results in fresh LN tissues to promote the MPM-FLIM technique for translation as an intravital tool for melanoma metastasis diagnostics.

From a morphological point of view, the FLIM-MPM adds an additional image contrast compared with the pure intensity data. For example, in this study, prominent features such as pleomorphism, ${ }^{38}$ atypical cells, and cellular mitosis were discerned. As the rate of mitosis is one of the factors used to diagnose and determine the stage of melanoma, ${ }^{39}$ this too is an important feature to look at. It is also known that the density of blood vessels is increased in metastatic tissue ${ }^{40-42}$ thus the ability to clearly distinguish blood vessels and the presence of erythrocytes, as demonstrated (e.g., Fig. 4), is beneficial and adds further support for MPM-FLIM as a diagnostic tool for this type of tissue.

To conclude, this study demonstrates that MPM-FLIM-based on autofluorescence has the potential to visualize melanoma metastasis in human SLN tissue by being able to detect malignant atypical cells, healthy lymphocytes, blood vessels, and erythrocytes in the LN. Higher contrast is observed in the FLIM images in comparison with MPM intensity images, providing complementary morphological information. Further experiments on fresh LN tissues, are required to validate the approach as a diagnostic tool for early staging of malignant melanoma, potentially providing rapid histopathological analysis in conjunct with SLN surgery.

\section{Disclosures}

The authors claim no conflicts of interest.

\section{Acknowledgments}

The authors would like to thank Shahin De-Lara, University of Gothenburg, for assisting in the deparaffinization procedure, and Roger Olofsson Bagge, University of Gothenburg, for contributing to the study design. Financial support was provided by the Swedish Research Council (No. VR 2015-05002), the Carl Trygger Foundation, and governmental funding under the ALF Agreement.

\section{References}

1. W. R. Zipfel, R. M. Williams, and W. W. Webb, "Nonlinear magic: multiphoton microscopy in the biosciences," Nat. Biotechnol. 21, 1369-1377 (2003).

2. S. W. Perry, R. M. Burke, and E. B. Brown, "Two-photon and second harmonic microscopy in clinical and translational cancer research," Ann. Biomed. Eng. 40, 277-291 (2012).

3. G. Pellacani et al., "The impact of in vivo reflectance confocal microscopy for the diagnostic accuracy of melanoma and equivocal melanocytic lesions," J. Invest. Dermatol. 127, 2759-2765 (2007).

4. T. Gambichler, V. Jaedicke, and S. Terras, "Optical coherence tomography in dermatology: technical and clinical aspects," Arch. Dermatol. Res. 303, 457-473 (2011). 
5. Z. Ying et al., "Elastic scattering spectroscopy for early detection of breast cancer: partially supervised Bayesian image classification of scanned sentinel lymph nodes," J. Biomed. Opt. 23(8), 085004 (2018).

6. S. Mert et al., "Kidney tumor staging using surface-enhanced Raman scattering," J. Biomed. Opt. 20(4), 047002 (2015).

7. M. Balu et al., "Distinguishing between benign and malignant melanocytic nevi by in vivo multiphoton microscopy," Cancer Res. 74, 2688-2697 (2014).

8. M. N. Pastore et al., "Non-invasive metabolic imaging of melanoma progression," Exp. Dermatol. 26, 607-614 (2017).

9. C. Chang, D. Sud, and M. Mycek, "Fluorescence lifetime imaging microscopy," Methods Cell Biol. 81, 495-524 (2007).

10. E. B. van Munster and T. W. J. Gadella, "Fluorescence lifetime imaging microscopy (FLIM)," in Microscopy Techniques, J. Rietdorf, Ed., pp. 143-175, Springer, Berlin, Heidelberg (2005).

11. J. R. Lakowicz et al., "Fluorescence lifetime imaging," Anal. Biochem. 202, 316-330 (1992).

12. X. F. Wang et al., "Fluorescence lifetime imaging microscopy (FLIM): instrumentation and applications," Crit. Rev. Anal. Chem. 23, 369-395 (1992).

13. W. Becker, "Fluorescence lifetime imaging- techniques and applications," J. Microsc. 247, 119-136 (2012).

14. P. P. Provenzano, K. W. Eliceiri, and P. J. Keely, "Multiphoton microscopy and fluorescence lifetime imaging microscopy (FLIM) to monitor metastasis and the tumor microenvironment," Clin. Exp. Metastasis 26, 357-370 (2009).

15. M. C. Skala et al., "In vivo multiphoton microscopy of NADH and FAD redox states, fluorescence lifetimes, and cellular morphology in precancerous epithelia," Proc. Natl. Acad. Sci. U.S.A. 104, 19494-19499 (2007).

16. C. Karimkhani et al., "The global burden of melanoma: results from the Global Burden of Disease Study 2015,” Br. J. Dermatol. 177, 134-140 (2017).

17. D. S. Rigel and J. A. Carucci, "Malignant melanoma: prevention, early detection, and treatment in the 21st century," Ca-Cancer J. Clin. 50, 215-236 (2000).

18. S. L. Wong et al., "Sentinel lymph node biopsy for melanoma: American Society of Clinical Oncology and Society of surgical oncology joint clinical practice guideline," J. Clin. Oncol. 30, 2912-2918 (2012).

19. S. E. L. Leong, "The role of sentinel lymph nodes in malignant melanoma," Surg. Clin. North Am. 80, 1741-1757 (2000).

20. F. Amersi and D. L. Morton, "The role of sentinel lymph node biopsy in the management of melanoma," Adv. Surg. 41, 241-256 (2007).

21. J. F. Gibbs et al., "Accuracy of pathologic techniques for the diagnosis of metastatic melanoma in sentinel lymph nodes," Ann. Surg. Oncol. 6, 699-704 (1999).

22. M. E. Valsecchi et al., "Lymphatic mapping and sentinel lymph node biopsy in patients with melanoma: a meta-analysis," J. Clin. Oncol. 29, 1479-1487 (2011).

23. W. R. Wrightson et al., "Complications associated with sentinel lymph node biopsy for melanoma," Ann. Surg. Oncol. 10, 676-680 (2003).

24. L. G. Wilke et al., "Surgical complications associated with sentinel lymph node biopsy: results from a prospective international cooperative group trial," Ann. Surg. Oncol. 13, 491-500 (2006).

25. D. Kantere et al., "Descriptive study of lymph nodes from melanoma patients using advanced laser scanning microscopy," Archives of Pathology and Laboratory Medicine, under review (2019).

26. S. Seidenari et al., "Multiphoton laser tomography and fluorescence lifetime imaging of melanoma: morphologic features and quantitative data for sensitive and specific noninvasive diagnostics," PLoS One 8, e70682 (2013).

27. E. Dimitrow et al., "Spectral fluorescence lifetime detection and selective melanin imaging by multiphoton laser tomography for melanoma diagnosis," Exp. Dermatol. 18, 509-515 (2009).

28. W. Becker et al., "Fluorescence lifetime imaging by time-correlated single-photon counting," Microsc. Res. Tech. 63, 58-66 (2004). 
29. I. Georgakoudi and K. P. Quinn, "Optical imaging using endogenous contrast to assess metabolic state," Аnпи. Rev. Biomed. Eng. 14, 351-367 (2012).

30. D. K. Bird et al., "Metabolic mapping of MCF10A human breast cells via multiphoton fluorescence lifetime imaging of the coenzyme NADH," Cancer Res. 65, 8766-8773 (2005).

31. M. C. Skala et al., "In vivo multiphoton fluorescence lifetime imaging of protein-bound and free nicotinamide adenine dinucleotide in normal and precancerous epithelia," J. Biomed. Opt. 12(2), 024014 (2007).

32. D. Li et al., "Two-photon excited hemoglobin fluorescence provides contrast mechanism for label-free imaging of microvasculature in vivo," Opt. Lett. 36, 834-836 (2011).

33. C.-H. Wu et al., "Imaging cytometry of human leukocytes with third harmonic generation microscopy," Sci. Rep. 6, 37210 (2016).

34. K. Koenig and I. Riemann, "High-resolution multiphoton tomography of human skin with subcellular spatial resolution and picosecond time resolution," J. Biomed. Opt. 8(3), 432-439 (2003).

35. F. Poulon et al., "Comparison between fresh and fixed human biopsies using spectral and lifetime measurements: fluorescence analysis using one and two photon excitations," in Int. Conf. Adv. Biomed. Eng., pp. 25-28 (2015).

36. X. Wang et al., "Effect of fixation and mounting on fluorescence lifetime of cellular autofluorescence," IEEE J. Sel. Top. Quantum Electron. 25, 7202606 (2019).

37. I. Saytashev et al., "Multiphoton excited hemoglobin fluorescence and third harmonic generation for non-invasive microscopy of stored blood," Biomed. Opt. Express 7, 3449-3460 (2016).

38. L. D. Su et al., "Sentinel lymph node biopsy for patients with problematic spitzoid melanocytic lesions," Cancer 97, 499-507 (2003).

39. S. D. Nathanson, R. Shah, and K. Rosso, "Sentinel lymph node metastases in cancer: causes, detection and their role in disease progression," Semin. Cell Dev. Biol. 38, 106-116 (2015).

40. R. H. Farnsworth et al., "Vascular remodeling in cancer," Oncogene 33, 3496-3505 (2014).

41. S. Y. Lee et al., "Changes in specialized blood vessels in lymph nodes and their role in cancer metastasis," J. Transl. Med. 10, 206-206 (2012).

42. Y. Murayama et al., "Precise detection of lymph node metastases in mouse rectal cancer by using 5-aminolevulinic acid," Int. J. Cancer 125, 2256-2263 (2009).

Jeemol James received her master's degree in optoelectronics and laser technology from the International School of Photonics, Cochin University of Science and Technology, India, in 2017. Currently, she is employed as a PhD student in the Biomedical Photonics Group, University of Gothenburg, Sweden. Her research area is in the application of the multiphoton microscope for quantitative biomedical applications, particularly focusing on fluorescence lifetime imaging and fluorescence correlation spectroscopy.

Despoina Kantere received her MD degree in 2003 from the University of Athens. She is presently working as a specialist in dermatology and venereology at Sahlgrenska University Hospital, Gothenburg, Sweden. She is a PhD student. Her research fields are skin tumors and two-photon fluorescence microscopy, as well as reflectance confocal microscopy.

Jan Siarov received his MD degree in 2010 from the University of Gothenburg. He is presently working as a specialist in pathology at Sahlgrenska University Hospital, Gothenburg, Sweden, subspecializing in dermatopathology.

Marica B. Ericson received her PhD in physical chemistry from Chalmers University of Technology and has had clinical research training as a postdoc at Sahlgrenska University Hospital. She is a principal investigator for the Biomedical Photonics Group, with an interdisciplinary track record ranging from optical spectroscopy to clinical applications. Her research focus is in advanced optical microscopy techniques for biomedical studies. In 2010, she became an associate professor in physics and has been a full professor in physical chemistry at the University of Gothenburg since 2018.

Biographies of other authors are not available. 